\title{
Fractographic study of transverse cracks in a fibre composite
}

\author{
TSUNG-YU PAN, RICHARD E. ROBERTSON*, FRANK E. FILISKO \\ Department of Materials Science and Engineering and Macromolecular Research Center, \\ The University of Michigan, Ann Arbor, Michigan 48109-2136, USA
}

\begin{abstract}
Transverse fracture of unidirectional fibre composites was studied in a model glass/epoxy composite in which $1 \mathrm{~mm}$-diameter rods had been used in place of fibres. The fracture surface resulting from transverse cracking in this model system was studied by scanning electron microscopy (SEM). The interaction of the crack with the epoxy matrix resin and the glass rods was the following: Cracks in the resin appeared to have effected a debonding at the glassmatrix interface before reaching the glass. The debonding then propagated along the interface and induced secondary cracks ahead of the primary debonding crack. The confluence of the secondary and primary cracks resulted in sharp ridges being formed on the matrix resin surface, produced by plastic deformation of the rigid epoxy resin. These appeared as a field of parabolic marks. Considering the brittleness of the resin, the amount of plastic deformation indicated by the ridges was astonishing. As the debonding continued around the glass rod, a transverse corrugated texture developed on the resin surface, again produced by plastic deformation. Finally, the cracks reentered the matrix from small patches of polymer adhering especially strongly to the glass surface. The overall fracture energy of transverse cracking of unidirectional fibre composites is suggested to consist, therefore, of the following elements in addition to crack propagation in the matrix resin: (a) the glass-resin debonding before the incoming cracks reach the glass, (b) the initiation of secondary cracks or debonds at the interface, (c) the plastic deformation in generating the ridges on the rigid resin surface, appearing both as the paraboloids and the transverse corrugation, and (d) cracking of the matrix reinitiated at the opposite side of the glass. The use of an enlarged glass reinforcement in this study provided a more direct observation of the properties of transverse crack propagation in composite materials than would have been possible with the small, roughly $10 \mu \mathrm{m}$ fibres.
\end{abstract}

\section{Introduction}

The low transverse tensile strength of unidirectional fibre laminae presents a major problem in the design of composite structures. The low transverse strength is due mainly to its being dominated by the matrix rather than the fibre. The transverse strength is less even than the strength of the matrix resin alone [1]. The reasons for this seem to involve a modest to weak bonding between the fibre and matrix and stress concentrations at the interface, arising from matrix shrinkage during cure and from mismatches in the thermal expansivities and moduli between the fibre and matrix resin [2]. Transverse cracking involves adhesive failure at the interface as well as cohesive failure of the resin [3]. A typical transverse cracking in a unidirectional fibre composite is shown in Fig. 1. The crack is seen to follow the interface to nearly the maximum extent possible. Thus, the interface between the fibre and the matrix is a crucial region in determining the low transverse strength.

Previous studies have revealed many features of transverse failure. Bailey et al. [4] observed directly a glass fibre/epoxy laminate in the scanning electron microscope (SEM) and showed that transverse cracks are formed by the coalescence and growth of fibre debonds. Microscopic studies of the fracture of glass/ polyester and graphite/epoxy laminae by Jones [5] and by Sinclair and Chamis [6] showed that transverse cracks are nucleated in regions of dense fibre packing and propagate along the interface preferentially by debonding. Chamis [7] found that the surface morphology of transverse cracks is heavily dependent on the interfacial bonding conditions and results in either cohesive or adhesive failure. Chai [8] indicated that this morphology depends largely on ply orientation and specimen geometry, at least for graphite/epoxy composites. Johannesson et al. [9] studied angle-ply specimens of graphite/epoxy failed in tension and found that the fibre-matrix interface debonded first and then the cracks propagate into the resin to create interleaving lamellae. Giare [10] investigated unidirectional glass fibre/epoxy composites in Mode II failure.

*Author to whom all correspondence should be addressed. 


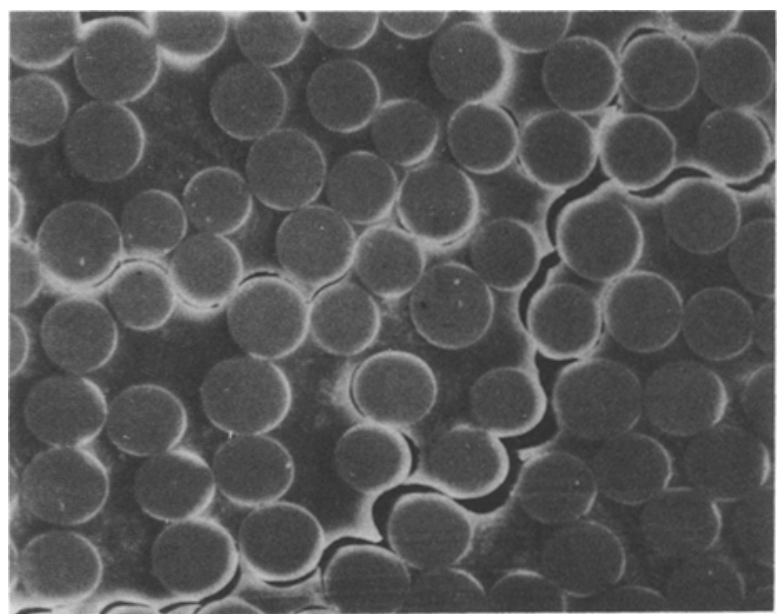

Figure I Transverse cracking in a typical unidirectional fibre composite. The diameters of fibres are $10 \mu \mathrm{m}$.

He suggested that the fracture energy is dissipated principally by the debonding of fibres from the matrix.

These studies indicate that transverse crack propagation generally involves debonding at the interface. Little is known, however, of how a transverse crack propagates along the interface. The purpose of this study was to investigate the mechanism of transverse crack propagation around the fibre in the interface region. To simplify the study, the usual $10 \mu \mathrm{m}$ fibres were replaced by $1 \mathrm{~mm}$ diameter glass rods. This allowed much easier inspection of the fracture morphology at the fibre-matrix interface. Because of the general scaling of the stress field, the results of this model study are expected to be directly applicable to that of fibre composites with normal size fibres.

\section{Experimental and analytical procedures}

\subsection{Materials}

The polymer matrix used in this study was an aminecured epoxy. This epoxy was D.E.R. 331 from Dow Chemical. It is a diglycidyl ether of bisphenol-A (DGEBA) with an epoxide equivalent weight of 182 to 190 , a molecular weight of 378 , and a viscosity in the range 11000 to $14000 \mathrm{cP}$ at $25^{\circ} \mathrm{C}$. The curing agent, also from Dow Chemical, was an experimental amine mixture (XPR-0544-010278-48-1, UN 1760) containing aliphatic and aromatic amines. This curing agent was recommended for its fast curing cycle and minimum residual stresses.

The cured resin has a Young's modulus of $3.4 \mathrm{GPa}$ $\left(500 \times 10^{3}\right.$ p.s.i. $)$, Poission's ratio of 0.35 , tensile strength of $76 \mathrm{MPa}\left(11 \times 10^{3}\right.$ p.s.i. $)$ and ultimate elongation of $4.5 \%$.

The reinforcement used was Corning Pyrex 7740 borosilicate glass rods $1 \mathrm{~mm}$ in diameter. The glass rods were first baked in a furnace at $540^{\circ} \mathrm{C}\left(1000^{\circ} \mathrm{F}\right)$ for $2 \mathrm{~h}$ to pyrolyse possible organic contamination, then cleaned in refluxing isopropyl alcohol for $1 \mathrm{~h}$, and finally dried and kept at $150^{\circ} \mathrm{C}$ in a vacuum oven until the specimens were made.

There was no sizing or coupling agent applied on the glass surface. It is a general opinion that there is an

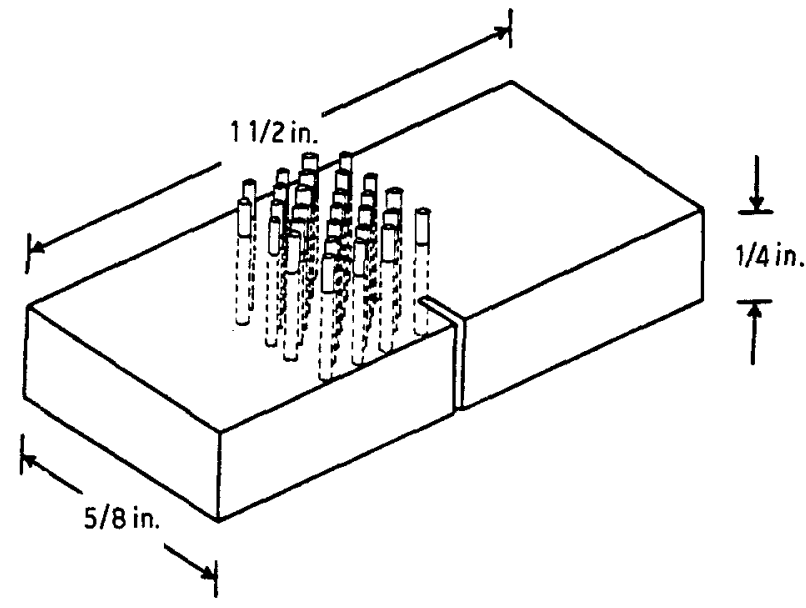

Figure 2 Specimen dimensions for the three-point bending fracture. The inclusions are $1 \mathrm{~mm}$ diameter glass rods arranged in an hexagonal pattern in DGEBA epoxy resin.

appropriate bonding between the glass and the epoxy matrix without coupling agent.

The glass rod has a Young's modulus of $62.7 \mathrm{GPa}$ $\left(9.1 \times 10^{6}\right.$ p.s.i. $)$, Poisson's ratio of 0.20 , and tensile strength of $3.4 \mathrm{GPa}\left(500 \times 10^{3}\right.$ p.s.i.).

\subsection{Specimen preparation}

Specimens were prepared in the form shown in Fig. 2. Glass rods, $32 \mathrm{~mm}$ long, were aligned vertically in a hexagonal pattern using a specially prepared fixture with $1.5 \mathrm{~mm}$ between the holes.

The epoxy resin was mixed with 17.2 p.h.r. curing agent (the stoichiometric ratio), and then poured into an aluminium foil mould with glass rods already aligned. The dimensions of the specimen were $38 \mathrm{~mm}$ long, $15.875 \mathrm{~mm}$ wide and $6.35 \mathrm{~mm}$ thick. The resin was cured at $50^{\circ} \mathrm{C}$ for $40 \mathrm{~min}$ and post-cured at $175^{\circ} \mathrm{C}$ for $2 \mathrm{~h}$ in an air-circulating oven. The specimens were oven-cooled to room temperature after curing.

\subsection{Fracture process}

The specimens were fractured by three-point bending. Notches were introduced in the specimens in the centre of an edge by cutting with a saw and sharpening with a razor blade. The bending fixture consisted of pins $4.76 \mathrm{~mm}$ in diameter with the supports separated by $25.4 \mathrm{~mm}$. Fracture was induced by an Instron 1137 Universal Testing Machine operated at a cross-head speed of $0.0508 \mathrm{~mm} \mathrm{~min}^{-1}$, which was equivalent to a strain rate of about $0.01 \mathrm{~min}^{-1}$. The notches were loaded so as to induce crack propagation in the crowd of glass rods more or less parallel with the axes of the rods.

\subsection{Scanning electron microscopy}

Prior to SEM examination, the fracture surfaces were coated with gold/palladium $(\mathrm{Au} / \mathrm{Pd})$ in a Technics Hummer VI Sputtering System. The SEM employed was a Hitachi S-520, operating with an accelerating voltage of $15 \mathrm{kV}$. In some cases, the normal to the fracture plane had to be tilted away from the incident electron beam toward the detector to enhance contrast from subtle topographic features. 


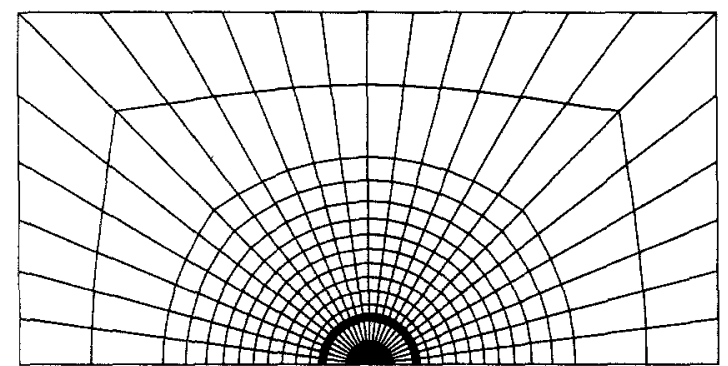

Figure 3 Finite element model for stress analysis.

\subsection{Finite element analysis}

A stress analysis was performed by finite element methods (FEM) [11] to calculate the stress field around the glass surface. The program was executed on the Michigan Terminal System (MTS) computer at the University of Michigan. The finite element grid used is shown in Fig. 3. The crack was placed in line with the rod axis (the equator). Because of symmetry about this plane, only the upper half of the specimen had to be generated. For simplicity, just a single glass inclusion of radius $r$ was modelled. In the absence of a crack, the entire lower boundary was constrained.

Only a linear elastic study was performed, and the problem was studied under plane strain conditions. A uniform tension traction was applied along the $y$-axis at a distance $7.5 r$ from the $x$-axis. The crack was assumed to be approaching the mid-line of the glass cylinder from the far right. The crack was simulated by freeing nodal points along the lower boundary. Three crack conditions were considered. The crack tip was locatd at $1.5 r, 1.0 r$, or $0.5 r$ away from the glass surface.

The material constants for the glass inclusion and the polymer matrix were assumed respectively to be 62.7 and $3.4 \mathrm{GPa}\left(9.1 \times 10^{6}\right.$ and $0.5 \times 10^{6}$ p.s.i. $)$ for the Young's modulus, and 0.20 and 0.25 for the Poisson's ratio.

\section{Experimental results}

Figs 4 and 5 give an overall view of the fracture surface resulting from a crack passing around a single

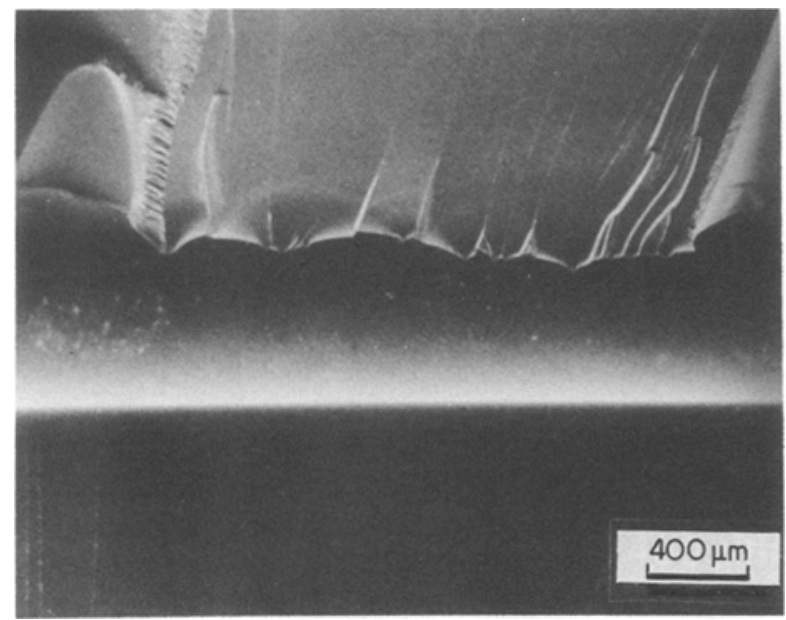

Figure 4 SEM fractograph of a transverse fracture surface containing the glass rod. Glass inclusion is aligned horizontally in the middle of the picture. The crack propagated from the bottom to the top.

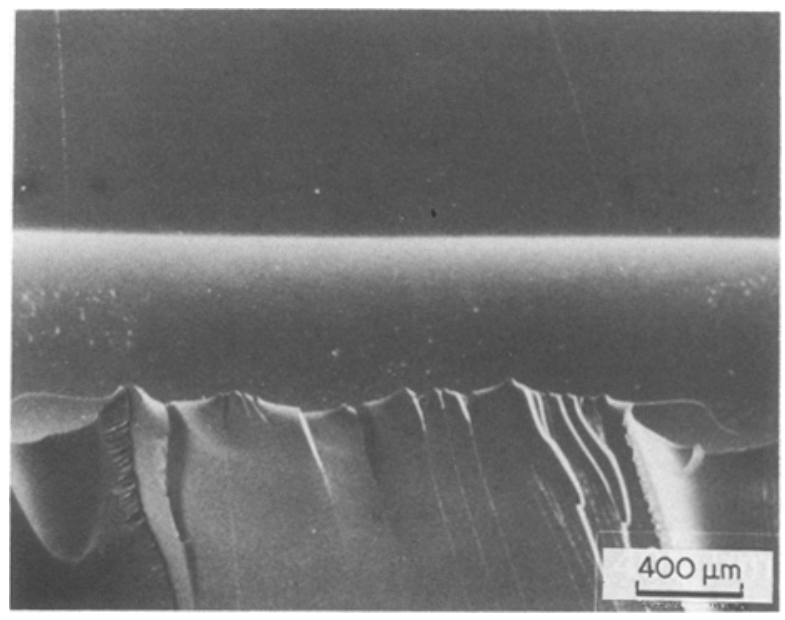

Figure 5 SEM fractograph of a transverse fracture surface, opposite to that in Fig. 4, containing polymer resin only. The crack propagated from the top to the bottom.

glass rod. Fig. 4 is the surface containing the glass rod; Fig. 5 is the matching surface containing polymer only. The width of the exposed glass surface is about twothirds of the $1 \mathrm{~mm}$ diameter of the glass rod, because the incoming crack surface was slightly above the axis of the glass rod. The crack passed upwards in Fig. 4 and downwards in Fig. 5. The crack in the matrix approached the glass rod with a straight boundary, went around the glass rod, and continued in the matrix resin on the other side of the rod. An irregular boundary with the rod surface was left along where the crack returned to the matrix, and the irregularity followed into the matrix as distinct lines parallel to the propagation direction in the matrix.

Fig. 6 is a magnified view of the region where the incoming crack reached the glass rod. The picture was taken by tilting the normal to the fracture plane away from the incident electron beam toward the detector by $75^{\circ}$. The crack propagated from the right as a flat plane until it reached the vicinity of the glass rod. Lines visible on the fracture surface are parallel to the propagation direction. These lines are the basic longitudinal texture [12-14], which are hypothesized to be the images left by the propagation of a finger-like

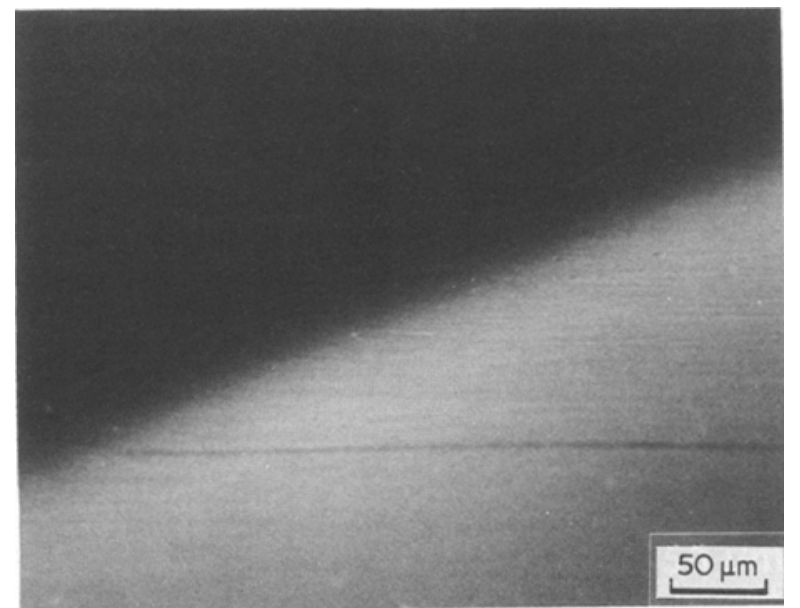

Figure 6 SEM picture showing the crack approaching the glass. The normal to the fracture surface was tilted towards the detector by $75^{\circ}$. The crack propagated from right to left 


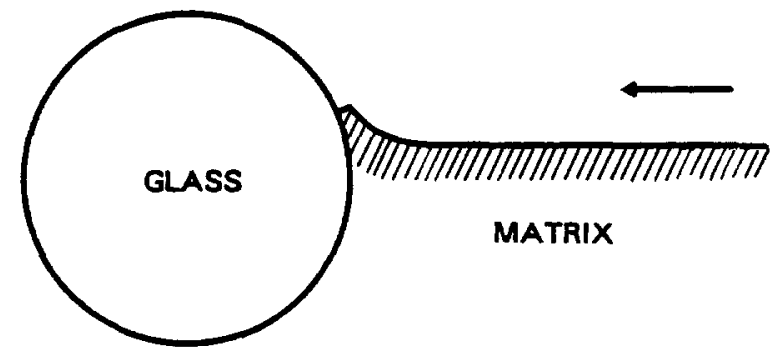

Figure 7 Schematic drawing of the fracture surface shown in Fig. 6

crack. The crack then rose slightly as it approached the glass surface, turning towards the tangential direction of the glass surface. This occurred at a distance 30 to $50 \mu \mathrm{m}$ from the glass, which was about 0.06 to 0.1 of the radius of the glass rod. Viewing the interface at even higher magnification, it is observed that the crack finally made a sharp turn perpendicular to the glass surface.

Fig. 7 shows a schematic illustration of how the incoming crack approached the glass rod. The interface region has been exaggerated to show the elevation of the fracture plane.

Figs 8 and 9 show the micrographs of the glassmatrix interface region. Again, Fig. 8 is the surface with the glass rod; Fig. 9 is the matching surface containing polymer only. The crack came from the upper-right corner in Fig. 8 and the lower-right corner in Fig. 9. The normal to the fracture surface was tilted toward the detector by $30^{\circ}$. Although the boundary between matrix and glass looks straight at low magnification in Figs 4 and 6, it is actually scalloped, as seen at higher magnification in Fig. 8. The matrix seems to be attached to the glass surface at the vertices of the scallop, as at A, but is seen to be separated from the glass in between, as in the vicinity of $B$.

On the polymer side of the glass-matrix interface (Fig. 9), many parabolic marks are visible. These marks, reaching a size around $2 \mu \mathrm{m}$ across, are oriented parallel to the crack propagation direction. Region $\mathrm{C}$, whose matching region is $\mathrm{A}$ in Fig. 8, has a mark extending from the scallop vertex toward the neigh-

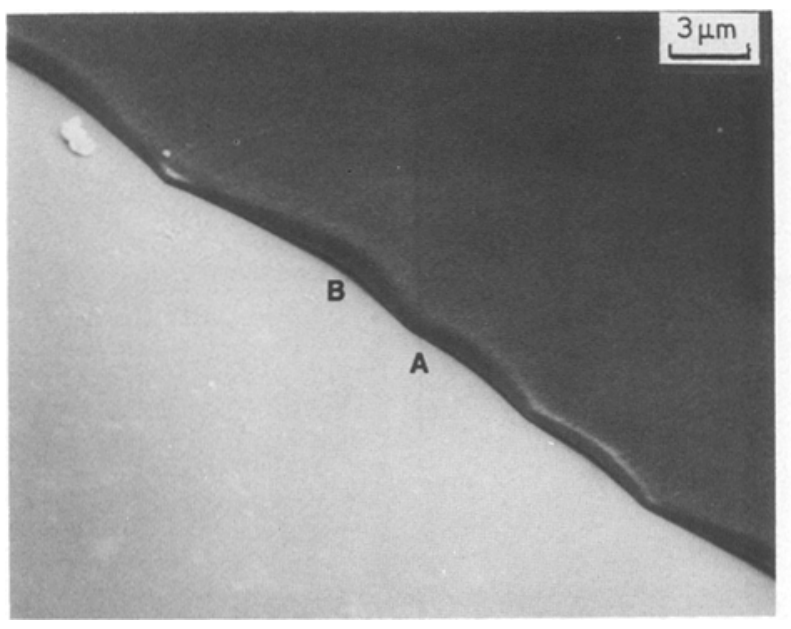

Figure 8 SEM fractograph of a fracture surface, containing the glass rod, at the boundary between the crack and the glass. The crack propagated from the upper right to the lower left.

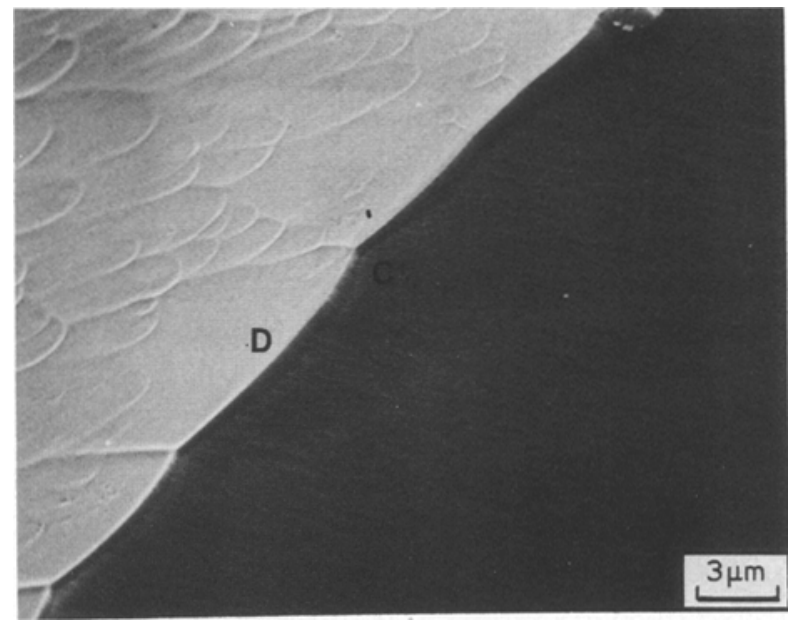

Figure 9 SEM fractograph of a fracture surface at the boundary between the crack and the glass, opposite to that in Fig. 8 and containing polymer only. The crack propagated from the lower right to the top left. Region $\mathrm{C}$ matched region $\mathrm{A}$ in Fig. 8, and region $\mathrm{D}$ matched region $\mathrm{B}$.

bouring paraboloid. Region $\mathrm{D}$, whose matching region is $\mathrm{B}$, shows neither paraboloids nor ridges.

Fig. 10 shows region $C$ of Fig. 9 at a magnification three times greater. The crack propagated from the lower-right corner towards the centre, then turned almost $90^{\circ}$ towards the glass-matrix interface, creating a step of about $0.5 \mu \mathrm{m}$ in height. The mark extending from a vertex of the scallop boundary to a parabola is better seen to be a ridge followed by three adjacent paraboloids.

A stereographic view of a group of paraboloids in Fig. 11 confirms that the edges of the paraboloids are sharp ridges, estimated to be $0.1 \mu \mathrm{m}$ in height. Fig. 12 is a schematic drawing of the boundary of parabolic marks. This indicates a significant degree of plastic deformation occurring in a brittle thermoset.

After the crack propagated around the glass rod to the opposite side, it continued in the polymer matrix. The surface of the fractured matrix where the crack leaves the glass is irregular, as shown in Fig. 4. One of the tips of this irregular boundary is shown in Figs 13 and 14. Fig. 13 is the surface with the glass rod, and

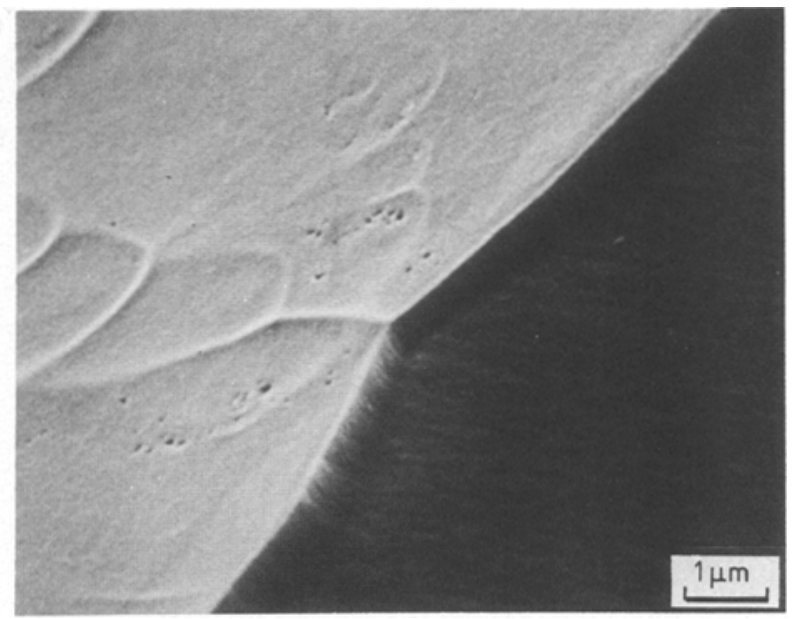

Figure 10 SEM fractograph of magnified region C in Fig. 9. The crack propagated from the right to the left. This micrograph shows the "step", "parabolic mark" and "ridge". 

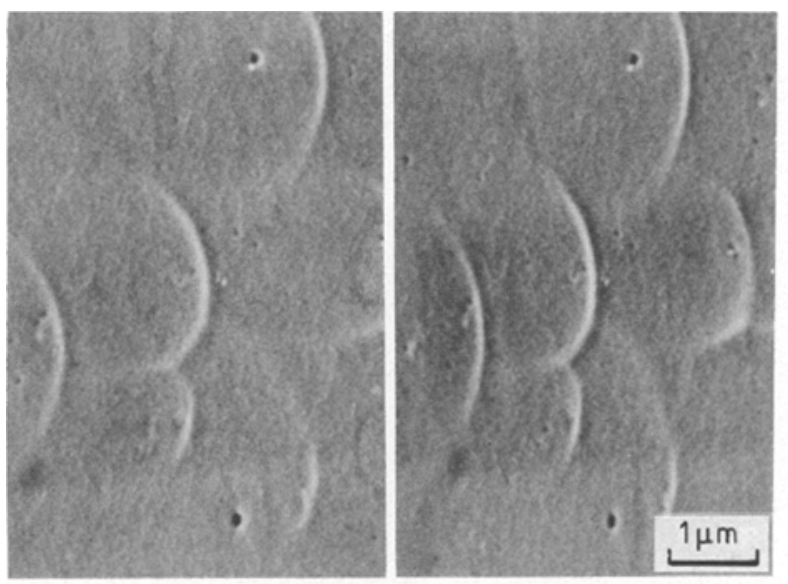

Figure 11 A stereograph of the parabolic marks.

Fig. 14 is the opposite surface containing polymer only. The primary crack came up from the bottom of Fig. 13 and down from the top of Fig. 14. After having followed the glass-matrix interface from one side of the rod to the other, the crack is seen to have been diverted back into the matrix at a $3 \mu \mathrm{m}$ diameter circular region. From there the crack fanned out into the matrix. The basic longitudinal texture towards the edges of the fan diverged even more and turned almost perpendicular to the edges of the fan and is directed back towards the glass-matrix interface. Gaps are visible between the edges of the fan and the glass surface in Fig. 13.

This $3 \mu \mathrm{m}$ diameter circular region was investigated from an angle near the horizontal of the glass surface, as shown in Fig. 15, by tilting the normal to the fracture surface toward the detector by $60^{\circ}$. The initiation centre attached to the glass surface is seen to be a very thin disc, about $0.4 \mu \mathrm{m}$ thick. The gap between the edges of the fan and the glass surface is quite apparent.

On viewing this vicinity on the polymer side of the fracture surface from an angle nearly parallel to the axis of the glass rod, a fine corrugated texture is visible, as shown in Fig. 16. This corrugated texture, with a periodicity around $0.5 \mu \mathrm{m}$, was perpendicular to the crack propagation direction. This corrugated texture seems to have developed only after the crack, having reached the top of the rod, was going down the other side, away from the stress normal. The parabolic marks had disappeared as the crack reached the top of the glass rod. The polymer surface at the top was featureless.

Fig. 17 shows the polymer side of the glass-matrix interface by looking along the axis of the glass rod at a magnification one-tenth of that in Fig. 16. The crack propagated from the right to the left. Many white marks, with long tails pointed away from the incoming crack, spread on the polymer surface of the interface,

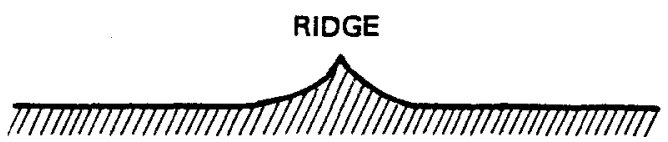

Figure 12 A schematic drawing of a "ridge", i.e. the boundary of parabolic marks.

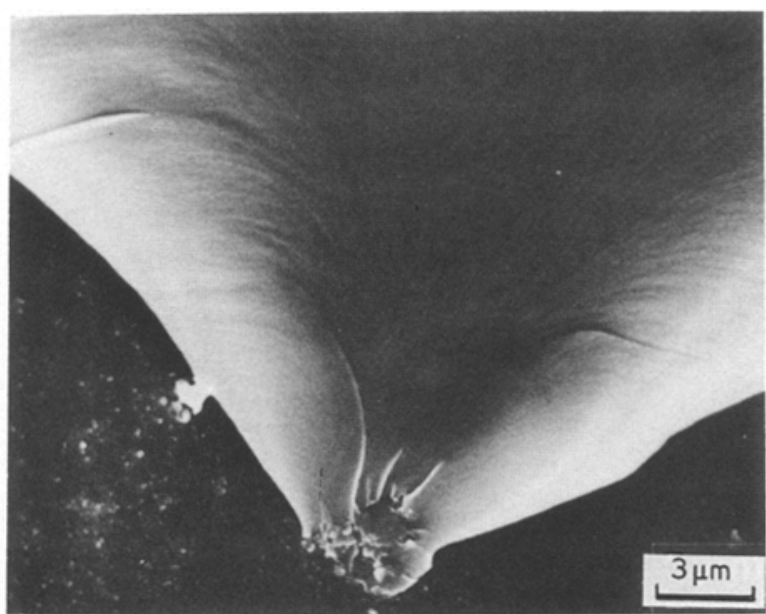

Figure 13 SEM fractograph of a fracture surface, containing a glass rod, with a new crack initiated on the matrix surface opposite the incoming crack. The crack propagated from the bottom to the top.

as meteors. These meteor-like marks further indicate the deformability of a brittle material.

\section{Analytical results}

The results of the stress analysis are shown in Figs 18 to 20 . The radial stress $\left(\sigma_{\mathrm{rr}}\right)$ at the glass-matrix interface is shown in Fig. 18; the maximum shear stress $\left(\tau_{\max }\right)$ is shown in Fig. 19; and the hoop stress $\left(\sigma_{\theta \theta}\right)$ is shown in Fig. 20. The relative stress intensity, i.e. the stress value divided by the applied stress $(\sigma)$, is plotted against the angle about the semicircle. The angle $0^{\circ}$ corresponds to the point facing the incoming crack; $90^{\circ}$ is at the top of the glass rod; and $180^{\circ}$ is opposite the incoming crack. The radial stress is the debonding force between the glass and the matrix, and is the most important parameter in this study. The maximum shear stress corresponds to shearing at the interface. The hoop stress at the interface involves stresses in the matrix but mainly in the glass.

In the absence of a crack, the radial stress $\sigma_{\mathrm{r}}$ has a maximum at $90^{\circ}$ and minima at $0^{\circ}$ and $180^{\circ}$. The maximum shear stress $\tau_{\max }$ has maxima at $45^{\circ}$ and

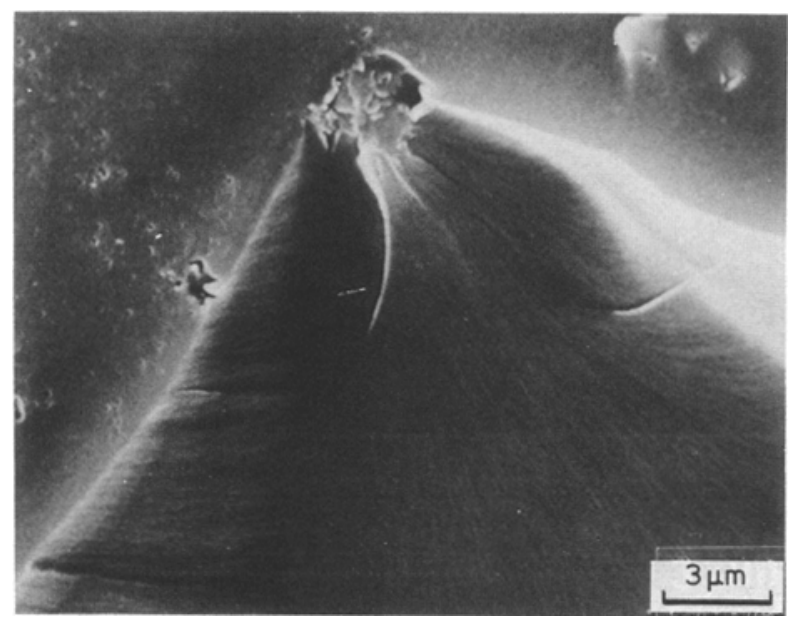

Figure 14 SEM fractograph of a fracture surface with a new crack initiated on the matrix surface opposite the incoming crack, opposite to that in Fig. 13 and containing polymer only. The crack propagated from the top to the bottom. 


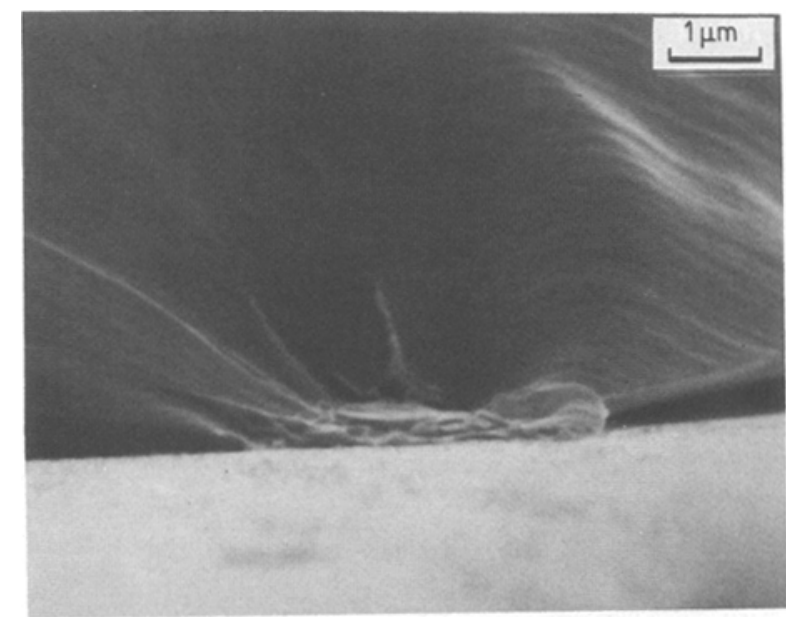

Figure 15 High-angle SEM fractograph of the image in Fig. 13 showing the crack initiation "disc" on the glass surface. The normal to the fracture surface was tilted towards the detector by $60^{\circ}$. The crack propagated from the bottom to the top.

$135^{\circ}$. The hoop stress $\sigma_{\theta \theta}$ has maxima at $0^{\circ}$ and $180^{\circ}$ and a minimum at $90^{\circ}$.

The glass surface facing the crack is exposed to much higher stress fields when the crack is present than when it is not. The hoop stress $\sigma_{\theta \theta}$ near the glass rod increases as the crack approaches the rod and, as expected, is a maximum in the vicinity of the approaching crack. In addition, the radial stress $\sigma_{T I}$ no longer has a minimum at $0^{\circ}$. It has the value of $2.8 \sigma$ at the glass-matrix interface, where $\sigma$ is the applied stress when the crack tip is $1.5 r$ from the glass surface. And this increases to $7.9 \sigma$ when the crack tip is $0.5 r$ from the glass surface. At higher angles, $\sigma_{I I}$ at the interface falls. At $90^{\circ}, \sigma_{r r}$ increases from $1.5 \sigma$ without a crack to only $3.4 \sigma$ when the crack tip is $0.5 r$ from the glass surface. Because of the high tensile stresses normal to the glass surface in the region around $0^{\circ}$ induced by the crack, debonding or cavitation may occur. The maximum of the shear stress $\tau_{\max }$ also occurs near the $0^{\circ}$ in the presence of the crack. This shear stress may cause shear deformation at the glassmatrix interface.

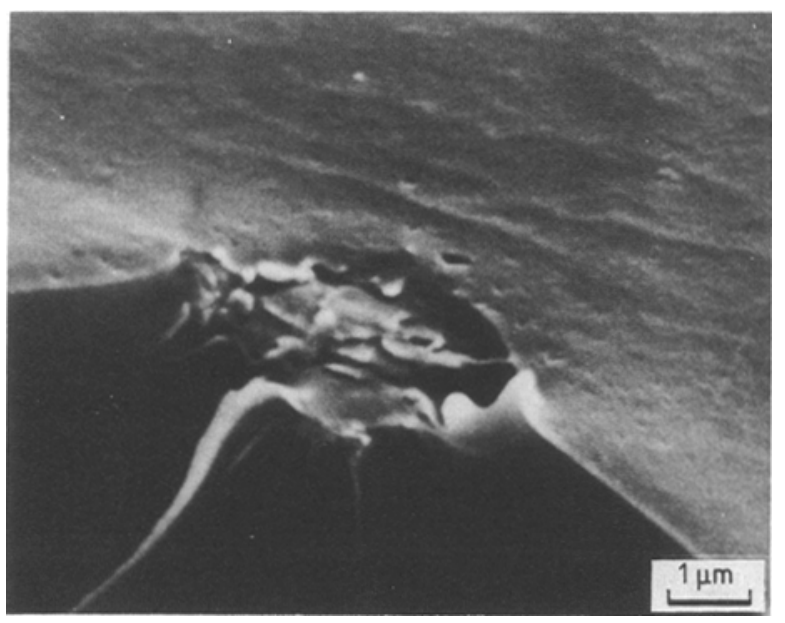

Figure 16 SEM fractograph showing the detail of the image around the crack initiation centre and the "corrugated texture" on the polymer side. The crack propagated from the upper right to the lower left.

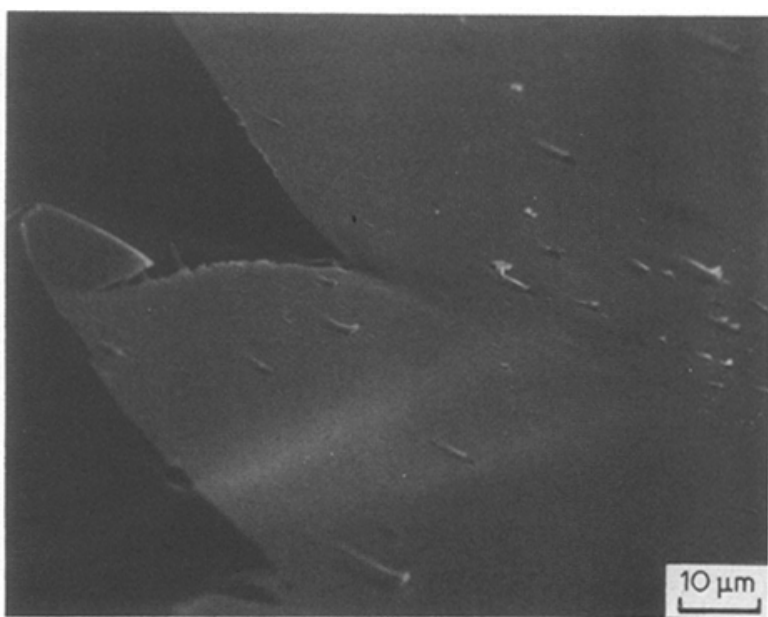

Figure 17 SEM fractograph of the polymer side of the interface, at a region similar to that in Fig. 14, viewed along the axis of the glass. The normal to the fracture surface was tilted towards the detector by $70^{\circ}$. The crack propagated from the right to the left.

The results of the finite element analysis for the non-crack case can be compared with the analytical solutions. The stresses inside and at the surface of a cylindrical inclusion embedded in an infinite slab of an otherwise homogeneous matrix material that is subjected to a stress perpendicular to the cylinder axis can be derived from Airy stress function. These have been shown to be $[15,16]$

$$
\begin{aligned}
& \sigma_{\mathrm{rr}} / \sigma=0.5\left(1+K_{1}\right)+0.5\left(1+K_{2}\right) \cos 2 \theta \\
& \sigma_{\theta \theta} / \sigma=0.5\left(1+K_{1}\right)-0.5\left(1+K_{2}\right) \cos 2 \theta \\
& \sigma_{\mathrm{r} \theta} / \sigma=-0.5\left(1+K_{2}\right) \sin 2 \theta
\end{aligned}
$$

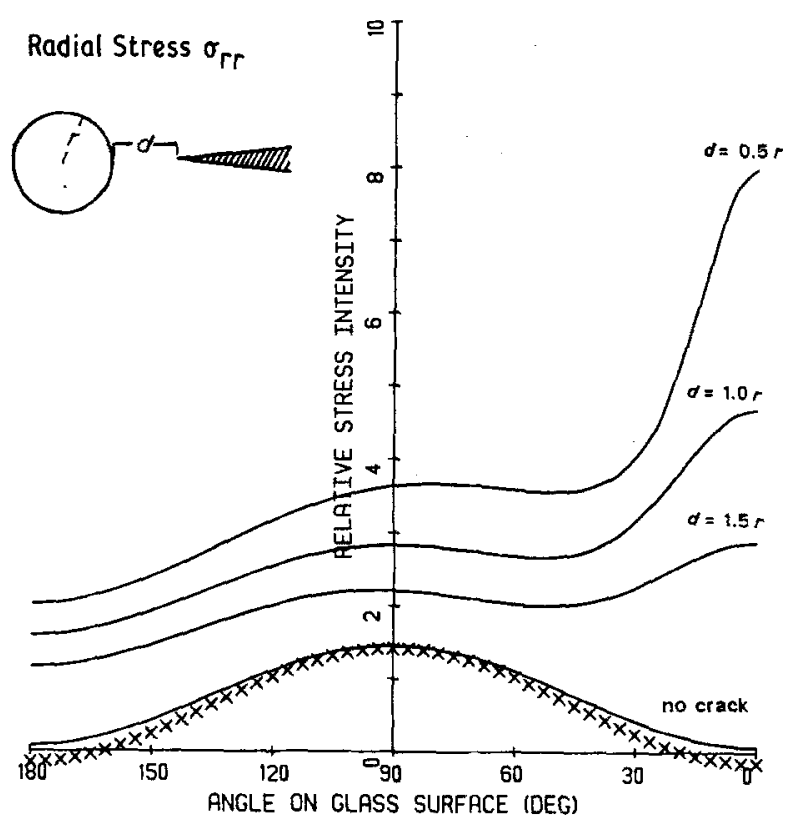

Figure 18 Radial stress distribution on the glass surface for unidirectionally loaded transverse crack problem. $d$ is the distance between the crack tip and the glass surface, $r$ is the radius of glass inclusion. "Relative stress intensity" is the stress value divided by the applied stress. The $0^{\circ}$ position is as the equator facing the crack. The $180^{\circ}$ position is at the opposite side. The $x \times \times$ curve represents the stress derived from analytic equations. 


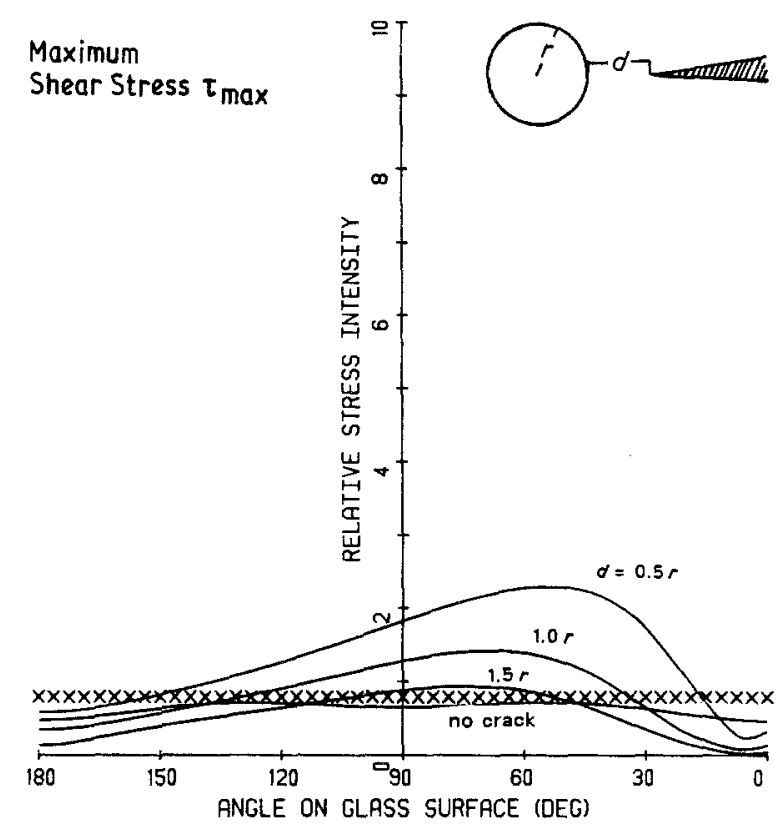

Figure 19 Maximum shear stress distribution on the glass surface for a unidirectionally loaded transverse crack problem. The symbols have the same meaning as in Fig. 18.

where the constants $K_{1}$ and $K_{2}$ are (for plane strain)

$$
\begin{aligned}
& K_{1}=\frac{E_{\mathrm{f}}\left(1-2 \mu_{\mathrm{m}}\right)\left(1+\mu_{\mathrm{m}}\right)-E_{\mathrm{m}}\left(1-2 \mu_{\mathrm{f}}\right)\left(1+\mu_{\mathrm{f}}\right)}{E_{\mathrm{f}}\left(1+\mu_{\mathrm{m}}\right)+E_{\mathrm{m}}\left(1-2 \mu_{\mathrm{f}}\right)\left(1+\mu_{\mathrm{f}}\right)} \\
& K_{2}=\frac{E_{\mathrm{f}}\left(1+\mu_{\mathrm{m}}\right)-E_{\mathrm{m}}\left(1+\mu_{\mathrm{f}}\right)}{E_{\mathrm{f}}\left(3-4 \mu_{\mathrm{m}}\right)\left(1+\mu_{\mathrm{m}}\right)+E_{\mathrm{m}}\left(1+\mu_{\mathrm{f}}\right)}
\end{aligned}
$$

and $E$ is the Young's modulus and $\mu$ is the Poisson's ratio. The subscripts $f$ and $m$ refer to the inclusion (fibre), and matrix, respectively. The maximum shear stress, $\tau_{\max }$ is then obtained from

$$
\tau_{\max }=0.5\left[\left(\sigma_{\mathrm{rr}}-\sigma_{\theta \theta}\right)^{2}+4 \sigma_{\mathrm{r} \theta}^{4}\right]^{1 / 2}
$$

The computed results from these equations are indicated by the $\times \times \times$ curves in Figs 18 to 20 . They are seen to be very close to those from the FEM. This is especially so for the radial stress, $\sigma_{\mathrm{rr}}$, which is the quantity of greatest interest to us.

Although the stress analysis is based on a linear elastic model, which means that the magnitudes of the stresses may not be exact, the analysis is able nonetheless to provide an adequate explanation of the observed debonding.

\section{Discussions}

Crack propagation in a material is determined by the stress state and the nature of the material. The usual variation of these leads to a significant range of crack path characteristics. The resulting fracture topography is, therefore, a record of the integration of material and mechanical factors that determine the crack path. A goal of this study has been to try to deconvolute these patterns into the material and mechanics factors, which might then suggest ways of improving properties.

\subsection{Parabolic marks}

One of the most surprising fractographic features found is the parabolic marking on the polymer surface where it had debonded from the glass. The parabolic marks are not the mirror image of the glass surface,

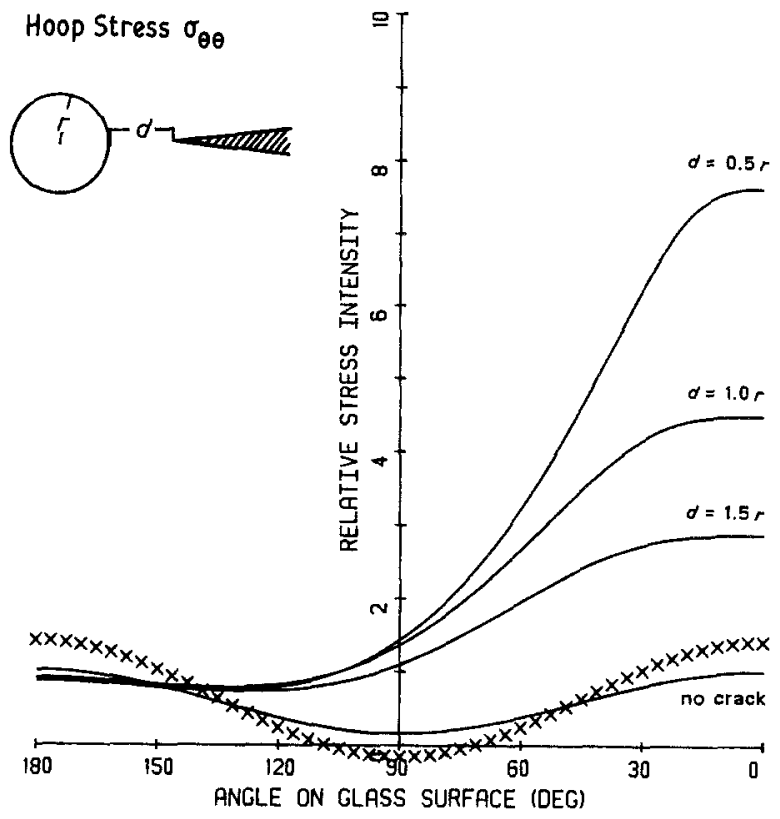

Figure 20 Hoop stress distribution on the glass surface for a unidirectionally loaded transverse crack problem. The symbols have the same meaning as in Fig. 18.

which is smooth. Rather, the marks are suggested to be the result of secondary crack or debond initiation ahead of the primary crack.

The secondary cracks themselves propagate uniformly outward as circles. On intersecting the primary crack front, as shown in Fig. 21, the parabolic figures are produced. The apex of the paraboloids are oriented in the direction from which the main crack has come. The initiation centre of the secondary crack appears as the focus of the parabola. This explanation was first proposed by Berry $[17,18]$, who discovered parabolicshaped regions of colour with light microscopy on the fracture surface of PMMA ruptured in tension. Berry suggested that secondary cracks are initiated from point flaws ahead of the primary fracture front. Further support for Berry's hypothesis was obtained recently

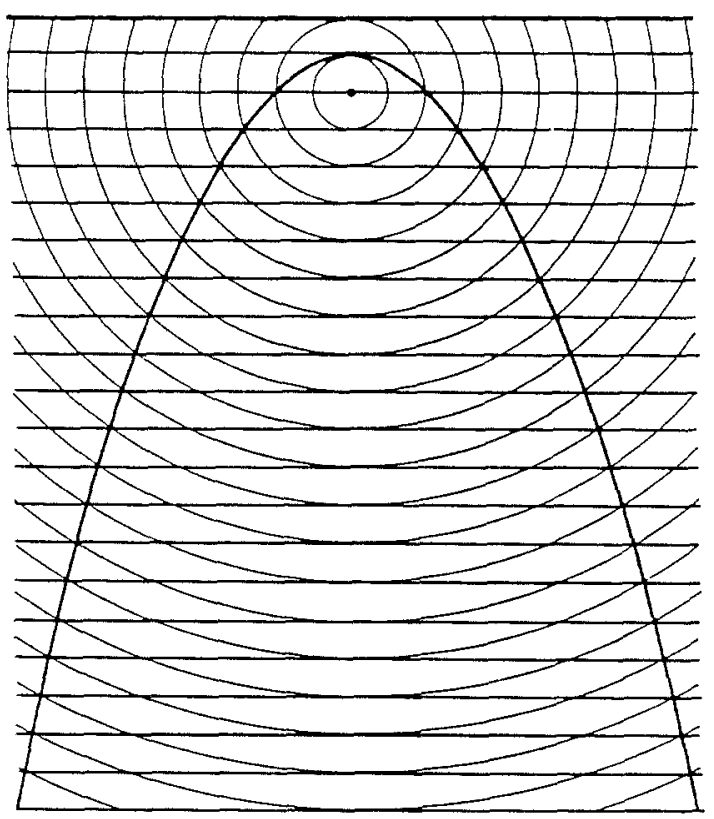

Figure 21 Generation of parabolic figures by the interaction of primary and secondary crack-fronts [18] 
by Robertson et al. [19] from a detailed SEM study of the fracture surface of a thermosetting polyester resin. The basic longitudinal texture, hypothesized to be the image of protruding fingers of a propagating crack front [12-14], was found to radiate outwardly from a foreign particle at the focus of the parabola. This texture then climbed and encountered the primary crack front at the edge of the paraboloid.

Parabolic marks are found also in metals, although a different terminology is used [20]. Such marks have been suggested to arise from a shearing and tearing of "dimples", which are caused by "microvoid" coalescence. Besides the slight similarity in appearance, the parabolic marks in metals and from the matrix debonding are similar in that they both arise from a superimposed shear stress. (As mentioned above, the paraboloids on the matrix resin surface occurred only where the glass surface was at an angle to the applied tensile stress, in a zone from where the crack first reached the glass surface and continued to a point below the top of the rod.)

The uniform size of the parabolic marks indicates that the fracture process at the interface is sequential. When the primary crack propagates along the interface, secondary cracks initiate at flaws at the interface ahead of the primary crack by the strong stress field at the crack tip.

The sharp ridges of the parabolic marks appear to have arisen from a plastic deformation of the resin induced by stress concentrations. The ridges have occurred when two secondary cracks or a secondary crack and the primary crack have coalesced. They would be the last of the matrix to be pulled from the glass surface as pairs of cracks come together. The height of the ridge is likely to depend on the deformability of the resin, the stress level at the interface, and the general adhesion of the resin to the glass surface.

There is no image of the parabolic marks on the glass surface. The modulus of the glass is about twenty times larger than that of the epoxy. The plastic deformation causing the parabolic marks on the polymer surface has had no effect on the glass.

\subsection{Debonding at the interface}

Debonding at the glass-matrix interface is suggested to occur before the crack tip reaches the glass. The two features that point to this are the observed separation between the matrix and the fibre and the fracture step as the crack in the matrix approached the glass.

The gap between the glass and the matrix is seen at the scalloped boundary in Fig. 8. If cavitation had not occurred before the crack arrived at the interface, the gap is unlikely to have developed. Similar evidence is found in region D of Fig. 9, the region matching the gap region in Fig. 8. A $3 \mu \mathrm{m}$ wide region between the glass-matrix boundary and the group of paraboloids is found here.

The step at the boundary between the primary crack and the glass in Figs 6 and 8 is suggested to have resulted from the interaction between the primary crack and the cavity at the interface. It has occurred during the final stage as the crack approached the glass. The sudden reorientation of the stress which has caused this is most easily explained by the loss of adhesion at the matrix-glass interface. The step size in Fig. 8 would be the distance between the crack tip and the interface when debonding occurred. As shown in Fig. 6, the crack began turning toward the tangential direction with respect to the glass surface as it approached the rod. This seems to have been due to the interaction of the stress field at the crack tip with the glass rod and has caused the crack to detour around the obstacle (the glass rod). The crack would have propagated continuously along this tangential direction if debonding had not occurred.

A related debonding mechanism has been suggested by Cook and Gordon [21]. Their prediction of debonding is for unidirectional fibre-reinforced composites when the stress is applied along the fibre axis and the plane of the crack is perpendicular to this axis. They have predicted a tensile component ahead of and in line with a running crack, which causes interfacial failure before the crack reaches the fibre. Their prediction arose from calculations of the stress distribution in a homogeneous body only, very close to a crack tip with a finite radius of curvature. They found that the tensile stress in line with the plane of the crack reaches a maximum not at the crack tip but about one radius in front of it. For a crack with a tip radius of $0.1 \mathrm{~nm}$, this tensile stress is 40 times larger than the applied load. When this region reaches the glass matrix interface, it would tend to cause debonding if the fibrematrix adhesion were less than the cohesive strength of the matrix. Cook and Gordon's mechanism has been confirmed by Owen [22], who investigated crack propagation in glass bead/epoxy composites by light microscopy and found that the spheres pulled free from the matrix in a zone ahead of the advancing crack. This mechanism has been used to explain the notch insensitivity of fibre composites.

Unfortunately, the radii of crack tips are rarely known, thus leading to uncertainty of the position and the value of in-plane tensile stresses. Even so, the present FEM stress analysis at the glass-matrix interface discussed in the previous section shows that $\sigma_{\mathrm{rr}}$ builds up very quickly around the $0^{\circ}$ position when a crack approaches the glass. From this analysis, it is reasonable to suggest that cavitation will occur at levels of $\sigma_{\mathrm{rr}}$ that are determined by the fibre-matrix adhesion.

The analysis in the present study has concentrated on the stress field at the interface instead of on the complicated calculation of the stress field through the whole sample as did Arridge [23] and Broutman and Agarwal [24]. Although our analysis used only a linear elastic model, the results seem to be adequate to explain the debonding phenomenon qualitatively.

\subsection{Morphology at the top of the inclusion}

There is no distinctive morphology on the polymer side of the interface at the top of the glass rod, despite the expectation that the applied stress would have resulted in high radial stresses at this position. The stress analysis shows, however, that $\sigma_{\text {rr }}$ is only $1.5 \sigma$ at the $90^{\circ}$ position in the absence of a crack and increases only gradually as the crack approaches the 
rod. Unlike the radial stress at $0^{\circ}$ in the presence of a crack, the magnitude of the radial stress at $90^{\circ}$ may not be high enough to induce any significant deformation.

\subsection{Morphology at the interface beyond the top}

As the crack continues to follow the interface beyond the top of the rod, a transversely oriented corrugation develops on the polymer surface. This texture is suggested to arise from a shear deformation and from slow crack propagation. The shear deformation induces the transverse corrugations. The slow rate of crack propagation damps out the crack fingering morphology.

Corrugated textures have also been seen on the fracture surface of metals, where it has been postulated to be the appearance of a new surface resulting from a "serpentine glide" on a series of favourably oriented near-parallel planes. Although there are no glide or slips plane as such in amorphous polymers, the shear deformation is possible and likely in this region of the curved glass-matrix interface.

The stress field local to the crack tip consists of two components: a tensile stress normal to the crack and a shear stress parallel to the crack [25]. The tensile stress tends to keep the crack propagating in a flat plane in a homogeneous material, and the shear component tends to deform the matrix to let the crack stay at the curved glass-matrix interface. The periodicity of the corrugated texture indicates the deformability of the matrix and the shear stress level at the interface.

Another phenomenon that suggests shear deformation at the polymer surface in this region is the meteor-like marks on the surface. These marks are believed to be the residue of the matrix resin that had adhered strongly to the glass surface and was pulled away from the rest of the matrix when the crack passed. The long tail arises from the shear deformation of the lifted polymer.

The slow rate of crack propagation along the interface in this region is suggested to occur because the stress field is increasingly attenuated as the crack moves away from a direction perpendicular to the applied load at the top of the glass rod. This behaviour was suggested also from the observations of Owen [22] in the study of a crack spreading on the surfaces of glass beads embedded in epoxy. Such slow crack growth is likely to damp out any instantaneous morphology arising from the propagating crack front, like crack fingering. For instance, Stork [26] and Donald and Kramer [27] studied the peel of Scotch tape. They found that the adhesive-air interface advances as a series of fingers at high peeling velocity, but at low peeling speeds, the crack or separation front is smooth and stable. Perturbations in its shape are damped out by the surface tension of the adhesive.

\subsection{Cracks in the matrix on the leaving side of the inclusion}

The cracks in the matrix on the leaving side of the glass rod appear to initiate at patches of polymer that are particularly well-adhered to the glass surface.
Somewhat surprisingly, the fracture planes on the leaving side are often at nearly the same latitude with respect to the glass cylinder as the incoming crack was on the other side of the rod. This may occur because both the entering and leaving cracks in the matrix are often near the equator of the circular inclusion. The reinitiation of the leaving cracks happens near the equator because the applied load is almost parallel to the glass-matrix interface in this region, which concentrates the stress on the adjacent matrix. The newly initiated crack then propagates perpendicular to the applied load and perpendicular to the initiation sites, as shown in Fig. 15.

The adhesion between the glass and the matrix is not uniform. The foci of the parabolic marks and the meteor-like texture are two examples of both poorer and better adhesion. The group of initiation sites of the crack in the matrix on the leaving side is another example. The interface regions adjacent to these initiation sites exhibit less adhesion, as is indicated by the observed gaps between the matrix and glass in Fig. 15. In addition to the localized reinitiation of fracture in the matrix, most of the original crack continues along the glass-matrix interface, thus generating this gap. The release of the matrix from the interface adjacent to the crack in the matrix results in a fairly complex local stress field. As is clearly visible in the basic longitudinal texture on the matrix fracture surfaces, this causes the initial crack tongue to fan out and even to reverse direction so that it travels back towards the interface, severing the resin as it goes.

Because the fracture in the matrix initiates at random sites on the glass surface, the boundary with the rod is ragged even at low magnification (Fig. 4). These random initiation sites are at different levels, and this causes the propagating cracks to be separated by steps [12-14]. If the original fracture surface is tilted relative to the axis of the glass rod, larger steps are expected on the leaving side. At larger angles, a lamellar texture can be induced [9, 28-31]. This mechanism can also explain the dependence of the morphology of a transverse crack on ply orientation as noted by Chai [8].

\subsection{Energy absorption by transverse cracks}

The fracture energy, the energy required to create the fracture surface, is related to the mechanism of crack propagation. Contributing to the fracture energy is the volume of material that is absorbing energy by undergoing plastic deformation and fracturing [32] The fracture energy for a transverse crack arises from several factors in addition to those resin variables mentioned by Lee [3]. These are (a) the glass-resin debonding before the incoming crack reaches the glass, which may stop fast crack propagation, (b) the initiation and growth of secondary cracks at the interface, which finally produces the paraboloids, (c) the plastic deformation in generating the ridges of the paraboloids, (d) the plastic deformation involved in the development of the transverse corrugation as the resin separates from the glass interface beyond the top of the inclusion, and (e) the crack reinitiation at the opposite side of the glass. It is not easy to determine 


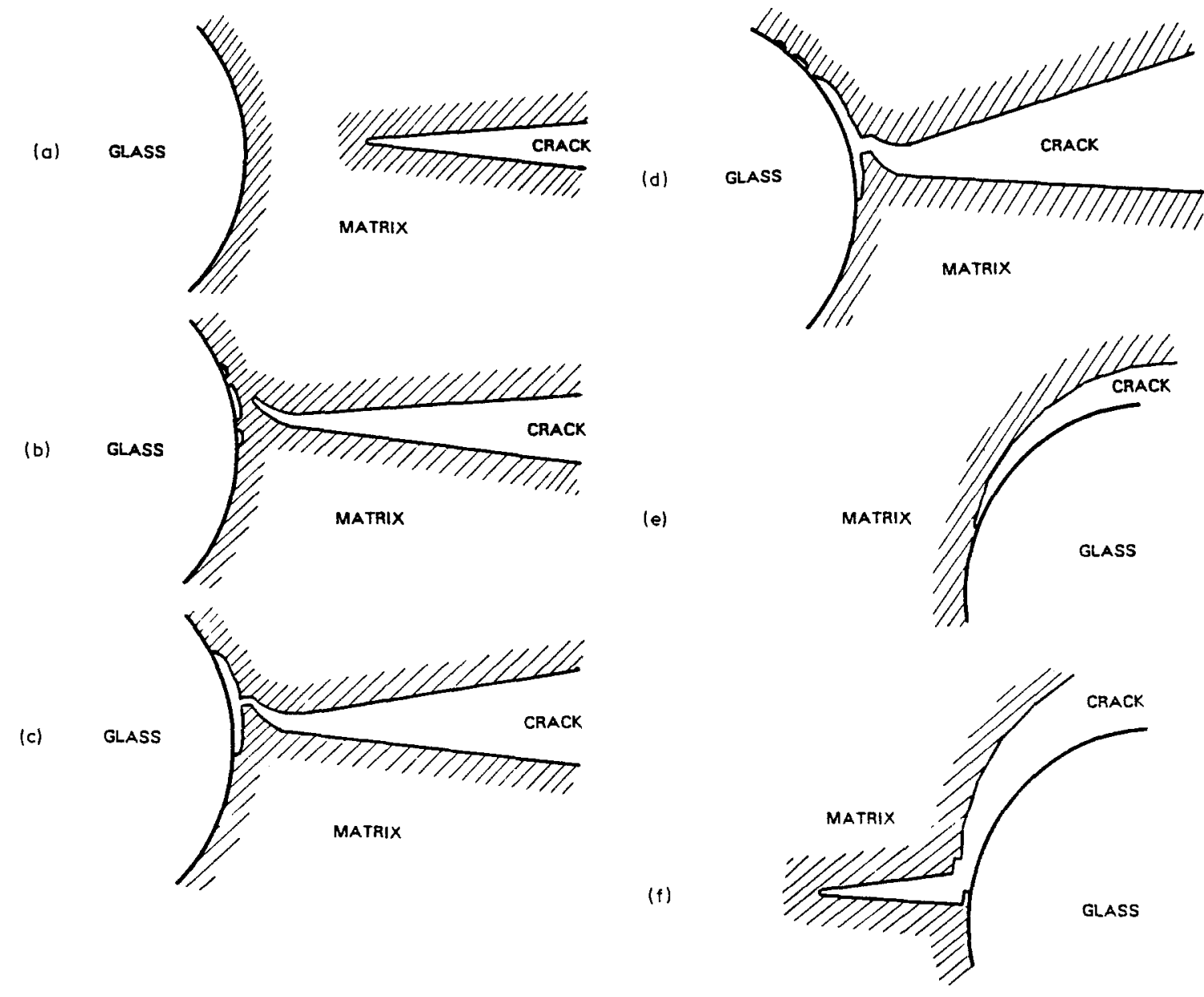

Figure 22 Proposed propagation properties of a transverse crack in a composite.

the relative importance of each of these. Nonetheless, it is likely that the fracture energy absorbing mechanisms must be throughly understood before attempts to improve the toughness against transverse cracks can be successful.

\subsection{Proposed properties for transverse cracking}

The properties for the propagation of a transverse crack in a glass-polymer composite are suggested to be the following and are shown schematically in Fig. 22:

(a) As the transverse crack in the matrix approaches the glass, the radial stress at the glass-matrix interface increases as a tensile stress.

(b) The crack turns towards the tangential direction with respect to the glass surface as it gets close to the glass. With the crack travelling above the equatorial plane, it will turn upwards. The radial stress has, by now, built up to very large values at the glass-matrix interface, which may cause debonding.

(c) The crack then continues to propagate along the glass-matrix interface. Secondary cracks are initiated ahead of the crack tip, and they are stretched to parabolic marks when the primary crack passes by.

(d) On passing beyond the top of the glass rod, the crack propagates more slowly due to the unfavourable load direction. A corrugated texture is generated on the polymer surface by the shear deformation.

(e) Near the equator of the glass rod, new cracks are initiated in the matrix from better-adhering patches of polymer and propagate into the matrix in a direction perpendicular to the load.

\section{Conclusions}

The use of an enlarged glass reinforcement in this study has provided a direct observation of the mechanism of transverse crack propagation in composite materials. Debonding occurs at the glassmatrix interface just ahead of the crack tip before the crack reaches the glass. Parabolic marks, identified as secondary cracks initiated ahead of the primary crack, arise from plastic deformation of the matrix between pairs of secondary cracks. A transverse corrugated texture, observed on the resin surface following separation of the interface beyond the top of the inclusion, is also generated by plastic deformation. Cracking of the matrix resin is reinitiated from small patches of polymer well-adhered to the glass surface. This behaviour was evident not only in glass-epoxy materials but also in glass-polyester materials investigated in a preliminary study. The mechanism of fracture energy absorption of a transverse crack propagating along the interface is suggested to arise from the combination of these fracture morphologies. It is expected that through studies like this that transverse cracking will be understood well enough for its fracture energy to be significantly increased. 


\section{Acknowledgements}

We wish to thank Professors Jwo Pan and Alan S. Wineman for their helpful advice on the stress analysis, and Ms Dorothy Babbington of Dow Chemical, Texas Division, for supplying the materials.

\section{References}

1. D. HULL, in "An Introduction to Composite Materials" (Cambridge University Press, Cambridge, 1981) pp. 145-54.

2. J. L. KARDOS, Polym. Preprint ACS 24 (1983) 185.

3. S. M. LEE, J. Mater. Sci. 19 (1984) 2278.

4. J. E. BAiley, P. T. CURTis and A. PARvizi, Proc, Roy. Soc. London A366 (1979) 599.

5. M. L. C. JONES, PhD thesis, University of Liverpool (1981).

6. J. H. SINCLAIR and C. C. CHAMIS, in Proceedings of 34th SPI/RP Annual Technical Conference, SPI, NY, 1979, paper 22-A.

7. C. C. CHAMIS, in "Composite Materials", Vol. 6, edited by E. P. Plueddemann (Academic, New York, 1974) pp. 40-3.

8. H. CHAI, Composites 15 (1984) 277.

9. T. JOHANNESSON, P. SJOBLOM and R. SELDEN, $J$. Mater. Sci. 19 (1984) 1171.

10. G. S. GIARE, Engng Fract. Mech. 1 (1984) 11.

11. N. KIKUCHI, in "Finite Element Methods in Mechanics" (Cambridge University Press, Cambridge, 1986).

12. R. E. ROBERTSON, V. E. MINDROIU and M. F. CHEUNG, Comp. Sci. Technol. 22 (1985) 197.

13. R. E. ROBERTSON, V. E. MINdroIU, Polym. Preprints $A C S$ 26(2) (1985) 115.

14. Idem, Polym. Engng Sci. 27 (1987) 55.

15. J. N. GOODIER, Trans. ASME 55 (1933) 39.

16. H. SCHUERCH, in "Mechanics of Composite Materials", Proceedings 5th Symposium on Naval Structure Mechanics, Philadelphia, Pennsylvania, 1967, edited by F. W. Wendt, H. Liebowitz and N. Perrone (Pergamon, Oxford, 1970) p. 583.
17. J. P. BERRY J. Appl. Phys. 33 (1962) 1741

18. Idem, in "Fracture Processes in Polymeric Solids", edited by B. Rosen (Interscience, New York, 1964) p. 195

19. R. E. ROBERTSON, T. Y. PAN and F. E. FILISKO, in preparation.

20. C. D. BEACHEM and D. A. MEYN, in "Electron Fractography", ASTM STP 436, (ASTM, Philadelphia, 1968) p. 59.

21. J. COOK and J. E. GORDON, Proc. Roy. Soc. London A282 (1964) 508.

22. A. B. OWEN, J, Mater, Sci. 14 (1979) 2521.

23. R. G. C. ARRIDGE Polym. Engng Sci. 15 (1975) 757.

24. L. J. BROUTMAN and B. D. AGARWAL, ibid. 14 (1974) 581 .

25. J. G. WILliaMS, in "Fracture Mechanics of Polymers" (Ellis Horwood, 1984).

26. S. T. STORK, PhD thesis, MIT (1968), quoted by A. S. Argon and M. Salama, Mater. Sci. Engng 23 (1976) 219.

27. A. M. DONALD and E. J. KRAMER, Phil. Mag. A43 (1981) 857 .

28. R, E. ROBERTSON and V. E. MINDROIU, $J$. Mater. Sci. 20 (1985) 2801

29. J. H. SINCLAIR and C. C. CHAMIS, NASA-TP-1081 (NASA, 1977)

30. G. E. MORRIS, in "Nondestructive Evaluation and Flaw Critically for Composite Materials", ASTM STP 696, edited by R. B. Pipes (ASTM, Philadelphia, 1979) p. 274.

31. J. AWERBUCH and H. T. HAHN, in "Fatigue of Fibrous Composite Materials", ASTM STP 723 (ASTM, Philadelphia, 1981) p. 243.

32. N. H. SUNG, T. J. JONES and N. P. SUH, J. Mater. Sci. $12(1977) 239$

Received 27 July

and accepted 23 October 1987 